\title{
THE ERO HOST GALAXY OF GRB 020127: IMPLICATIONS FOR THE METALLICITY OF GRB PROGENITORS
}

\author{
E. Berger, ${ }^{1,2,3}$ D. B. Fox, ${ }^{4}$ S. R. Kulkarni, ${ }^{5}$ D. A. Frail, ${ }^{6}$ and S. G. Djorgovski ${ }^{5}$ \\ Received 2006 September 5; accepted 2007 January 21
}

\begin{abstract}
We present optical and near-IR observations of the host galaxy of GRB 020127 , for which we measure $R-$ $K_{s}=6.2 \mathrm{mag}$. This is only the second GRB host to date classified as an ERO. The spectral energy distribution (SED) is typical of a dusty starburst galaxy, with a redshift $z \approx 1.9$, a luminosity $L \approx 5 L^{*}$, and an inferred stellar mass of $M_{*} \sim 10^{11}-10^{12} M_{\odot}$, two orders of magnitude more massive than typical GRB hosts. A comparison to the $z \sim 2$ massmetallicity $(M-Z)$ relation suggests that the host metallicity is about $0.5-1 Z_{\odot}$. This result shows that at least some GRBs occur in massive, metal-enriched galaxies, and that the proposed low-metallicity bias of GRB progenitors is not as severe as previously claimed. Instead, we propose that the blue colors and sub- $L^{*}$ luminosities of most GRB hosts reflect their young starburst populations. This explanation also accounts for the prevalence of low-redshift GRBs in low-mass galaxies, since star formation activity shifts from high- to low-mass galaxies as a function of decreasing redshift ("downsizing"). Thus, the low-metallicity bias claimed for $z \lesssim 0.2$ GRB hosts is likely a secondary effect, which reflects the $M-Z$ relation, and consequently GRBs and their hosts may serve as a reliable tracer of cosmic star formation activity.
\end{abstract}

Subject headings: galaxies: starburst — gamma rays: bursts

\section{INTRODUCTION}

Observations of the host galaxies of long-duration $\gamma$-ray bursts (GRBs) have led to the general consensus that they are faint and blue. This has been interpreted as evidence for intense star formation activity, as well as low metallicity and stellar mass (Fynbo et al. 2003; Le Floc'h et al. 2003; Christensen et al. 2004; Fruchter et al. 2006; Stanek et al. 2006). In particular, a comparison of $z \lesssim 0.2$ GRB hosts to a large sample of Sloan Digital Sky Survey (SDSS) galaxies suggests that GRBs occur preferentially in lowmetallicity galaxies with $Z \sim 0.1-0.5 Z_{\odot}$ (Stanek et al. 2006). These authors also propose an upper metallicity cutoff of about $0.15 Z_{\odot}$ for typical GRBs (i.e., those with $E \sim 10^{51} \mathrm{erg}$ ). At higher redshifts, $z \sim 0.5-1$, a comparison to the host galaxies of GOODS supernovae indicates that GRBs tend to occur in lower luminosity galaxies (by about $1 \mathrm{mag}$ ) and therefore presumably at lower metallicity (Fruchter et al. 2006). Finally, metallicities measured from afterglow absorption spectra (at $z>2$ ) span a range of $Z \sim 0.05$ $0.5 Z_{\odot}$ (e.g., Berger et al. 2006a; Prochaska 2006). Theoretical studies have also argued for "low" metallicity of GRB progenitors (MacFadyen \& Woosley 1999), although we stress that the definition is not well quantified, and black hole remnants can apparently be formed even above solar metallicity (Heger et al. 2003).

These observations raise two crucial and related questions: (1) Is there in fact a low-metallicity bias for GRB progenitors, in both an absolute and a relative sense? (2) Can GRBs and their host galaxies be used as a representative tracer of star formation at high redshift $(z \gtrsim 1)$ ? The answer to these questions is of great importance given the unique ability of GRBs to probe the

\footnotetext{
1 Observatories of the Carnegie Institution of Washington, Pasadena, CA 91101.

2 Princeton University Observatory, Princeton, NJ 08544.

3 Hubble Fellow.

4 Department of Astronomy and Astrophysics, Pennsylvania State University, University Park, PA 16802.

5 Division of Physics, Mathematics, and Astronomy, California Institute of Technology, Pasadena, CA 91125.

${ }^{6}$ National Radio Astronomy Observatory, Socorro, NM 87801.
}

interstellar medium and star-forming environments of galaxies over a wide redshift range, extending to $z>6$ (Berger et al. $2006 \mathrm{~b}$ ). It is also crucial to understand the effect of metallicity in light of the redshift evolution of the average metallicity and mass function of galaxies (e.g., Savaglio et al. 2005; Erb et al. 2006). Equally important, the metallicity range for GRB progenitors impacts our understanding of the progenitor population and GRB formation scenarios (MacFadyen \& Woosley 1999; Heger et al. 2003).

The prevalence of GRBs in blue galaxies may also reflect an observational bias against dusty galaxies due to obscuration of the optical afterglow. Two lines of evidence suggest that this may not be a significant problem. First, the host galaxies of the "optically dark" GRBs (those with clear evidence for dust obscuration in the optical band) are typically not redder than the hosts of optically bright GRBs (Berger et al. 2003; Le Floc'h et al. 2003). A notable exception is GRB 030115, with a dust-reddened afterglow and a host galaxy with $R-K \approx 5.4$ mag (Levan et al. 2006). Second, radio, submillimeter, and IR observations have not led to a preferential detection of the dark GRB hosts (Barnard et al. 2003; Berger et al. 2003; Le Floc'h et al. 2006), despite an expectation that these galaxies should have obscured star formation. Conversely, the host galaxies with long-wavelength detections (and hence obscured star formation) have blue colors typical of the GRB host population as a whole (Berger et al. 2003). Thus, the lack of a clear difference in the host properties of dark and bright GRBs suggests that obscuration of the optical afterglow does not lead to a strong observational bias.

Here we present optical and NIR observations of GRB 020127 , which reveal that the host galaxy is an extremely red object (ERO) with $R-K_{s}=6.2 \mathrm{mag}$. The GRB position is obtained from $\mathrm{X}$-ray and radio observations of the afterglow. The host SED is best fit by an obscured starburst galaxy template at $z \approx 1.9$, with a stellar mass ${ }^{7}$ of $\sim 10^{11}-10^{12} M_{\odot}$ and an inferred metallicity (Erb et al. 2006) of about $0.5-1 Z_{\odot}$, suggesting that GRB progenitors

\footnotetext{
7 We use the standard cosmology with $H_{0}=71 \mathrm{~km} \mathrm{~s}^{-1} \mathrm{Mpc}^{-1}, \Omega_{m}=0.27$, and $\Omega_{\Lambda}=0.73$.
} 
can in fact exist at near-solar metallicity and may occur in the most massive galaxies at $z>1$.

\section{AFTERGLOW AND HOST GALAXY OBSERVATIONS}

GRB 020127 was discovered by the High Energy Transient Explorer II satellite on 2002 January 27.875 UT, with a positional accuracy of $8^{\prime}$ radius (Ricker et al. 2002). The burst duration and fluence in the $2-400 \mathrm{keV}$ band are $18 \mathrm{~s}$ and $2.7 \times 10^{-6} \mathrm{erg} \mathrm{cm}^{-2}$ (Sakamoto et al. 2005).

Optical observations did not uncover an afterglow candidate, with $R, I>19.5 \mathrm{mag}$ at 4.4 and $8.2 \mathrm{hr}$ after the burst, respectively (Lamb et al. 2002), and $R>21.5 \mathrm{mag}$ at $3.1 \mathrm{hr}$ over $75 \%$ of the error circle (Castro Cerón et al. 2002). The Galactic extinction in the direction of GRB 020127 is low, $E(B-V)=0.048 \mathrm{mag}$ (Schlegel et al. 1998).

\subsection{X-Ray, Radio, and Optical/NIR Imaging}

We initiated two $10 \mathrm{ks}$ observations with the Chandra X-Ray Observatory, 4.14 and 14.64 days after the burst, to identify the fading X-ray afterglow. The data were obtained with the Advanced CCD Imaging Spectrometer (ACIS) and reduced and analyzed using the CIAO software package. ${ }^{8}$ A comparison of the two epochs reveals three fading sources not associated with bright stellar counterparts. Of these, only one is detected in both epochs and has faded with high significance, with 2.6 and 1.0 count $\mathrm{ks}^{-1}$ (0.3-7 keV), respectively. Using a Galactic column of $3 \times$ $10^{20} \mathrm{~cm}^{-2}$ (Schlegel et al. 1998) and a photon index of $1.5 \pm$ 0.4 , we find fluxes of $(5.5 \pm 0.1) \times 10^{-14}$ and $(2.1 \pm 0.7) \times$ $10^{-14} \mathrm{erg} \mathrm{cm}^{-2} \mathrm{~s}^{-1}$, respectively, corresponding to a power-law decay rate, $F_{\nu} \propto t^{-0.8}$, typical of GRB afterglows. The position of the X-ray afterglow candidate is $\alpha=08^{\mathrm{h}} 15^{\mathrm{m}} 01.42^{\mathrm{s}}, \delta=$ $+36^{\circ} 46^{\prime} 33.9^{\prime \prime}$ (J2000), with an uncertainty of about $1^{\prime \prime}$ in each coordinate.

We observed this source with the Very Large Array $\left(\mathrm{VLA}^{9}\right)$ at a frequency of $8.46 \mathrm{GHz}$ on 2002 February 14.20, 16.23, 21.97, and March 18.10 UT. The data were processed using AIPS. In the first observation we detect an object with a flux of $222 \pm 63 \mu \mathrm{Jy}$, coincident with the X-ray position at $\alpha=08^{\mathrm{h}} 15^{\mathrm{m}} 01.42^{\mathrm{s}}, \delta=$ $+36^{\circ} 46^{\prime} 33.45^{\prime \prime}(\mathrm{J} 2000), \pm 0.03^{\prime \prime}$ in each coordinate. We note that the systematic uncertainty between the radio position, which is tied to the International Celestial Reference System (ICRS), and the X-ray and optical (see below) positions is about $0.3^{\prime \prime}$ (see Bloom et al. 2002 for a full discussion). Subsequent observations reveal that the object has faded, with $3 \sigma$ limits of $150 \mu \mathrm{Jy}$ (February 16.23 and 21.97) and $65 \mu \mathrm{Jy}$ (March 18.10), suggesting that this is the radio afterglow of GRB 020127.

Optical observations were obtained with the Large Format Camera (LFC) on the 200 inch $(5 \mathrm{~m})$ telescope at the Mount Palomar Observatory on 2002 February 4.29 and 6.34 UT for a total of $4300 \mathrm{~s}\left(g^{\prime}\right), 2400 \mathrm{~s}\left(r^{\prime}\right)$, and $1800 \mathrm{~s}\left(i^{\prime}\right)$. The images were biassubtracted, flat-fielded, and co-added using IRAF, and photometry was performed relative to SDSS. At the position of the X-ray and radio afterglows we detect a faint object with $i^{\prime}=23.89 \pm$ $0.13 \mathrm{mag}, r^{\prime}>23.5 \mathrm{mag}$, and $g^{\prime}>25.8 \mathrm{mag}$; limits are $2 \sigma$ and magnitudes are in the AB system. We identify this object as the host galaxy of GRB 020127. Further observations were obtained with the Echellete Spectrograph and Imager (ESI) on the Keck II $10 \mathrm{~m}$ telescope on 2002 March 13.28 UT in $R(1500 \mathrm{~s})$ and on

\footnotetext{
8 See http://cxc.harvard.edu/ciao.

9 The VLA is operated by the National Radio Astronomy Observatory, a facility of the National Science Foundation operated under cooperative agreement by Associated Universities, Inc.
}

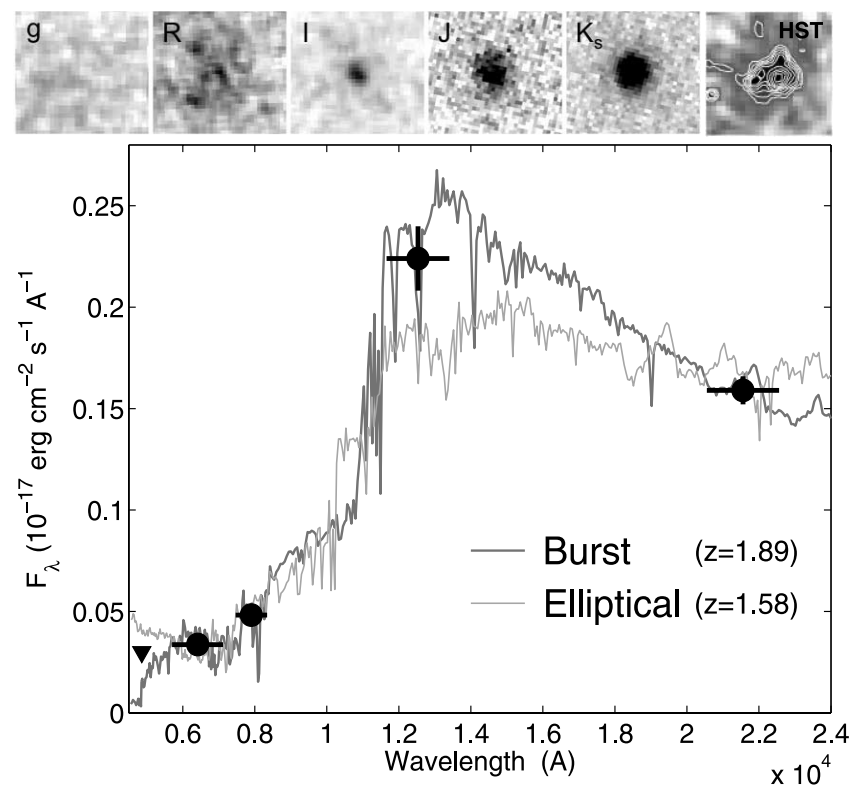

FIG. 1.- Spectral energy distribution of the host galaxy of GRB 020127 along with the best-fit starburst and elliptical galaxy models. The combination of a blue $J-K_{s}$ color and a deep upper limit in the $g$ band indicates that the starburst template is a much better fit compared to an elliptical galaxy. The top panel shows the host galaxy in each of the five observed bands.

2003 February 28.36 UT in $I(600 \mathrm{~s})$. The data were reduced as described above. We measure $I=23.56 \pm 0.10 \mathrm{mag}$ and $R=$ $24.73 \pm 0.15 \mathrm{mag}$ (Vega).

NIR observations in $K_{s}$ and $J$ were obtained with the NearInfrared Camera (NIRC) on the Keck I 10 m telescope on 2002 October 13.63 and November 17.48 UT, respectively, for a total of $1080 \mathrm{~s}$ in each filter. The individual frames were dark-subtracted, flat-fielded, and corrected for bad pixels and cosmic rays using custom IRAF routines. Photometry was performed relative to the standard star Feige 16. The host galaxy has $J=20.37 \pm 0.10$ mag and $K_{s}=18.54 \pm 0.05 \mathrm{mag}$ (Vega). Optical/NIR images of the host are shown in Figure 1. The position of the host galaxy, determined relative to $\mathrm{SDSS}$, is $\alpha=08^{\mathrm{h}} 15^{\mathrm{m}} 01.35^{\mathrm{s}}, \delta=+36^{\circ} 46^{\prime} 34.66^{\prime \prime}$ (J2000), $\pm 0.1^{\prime \prime}$ in each coordinate. This is fully consistent with the X-ray afterglow position and is about $1.4^{\prime \prime} \pm 0.6^{\prime \prime}$ from the radio afterglow position. Given a source density of objects brighter than $K_{s}=18.54$ mag of about $1.4 \times 10^{4} \mathrm{deg}^{-2}$ (Kochanek et al. 2001 ), the probability of chance coincidence is only about $7 \times 10^{-3}$.

Finally, on 2002 April 6 UT we observed the host galaxy with the Hubble Space Telescope (HST) using the Space Telescope Imaging Spectrograph (STIS) as part of program GO 9180 (PI: Kulkarni). A total of $4868 \mathrm{~s}$ were obtained with the CL filter. We processed and combined the individual exposures using the IRAF task drizzle (Fruchter \& Hook 2002), with pixfrac $=0.8$ and pixscale $=0.5$. At the position of the afterglow we detect an extended object with an $\mathrm{AB}$ magnitude of $24.8 \pm 0.1 \mathrm{mag}$ (Fig. 1).

\subsection{Optical Spectroscopy}

We obtained optical spectra of the host galaxy using ESI on four separate occasions. The data were reduced using custom IRAF routines to bias-subtract, flat-field, and rectify the 10 individual echelle orders. Sky subtraction was performed using the method and software described in Kelson (2003). Wavelength calibration was performed using $\mathrm{CuAr}$ and $\mathrm{HgNeXe}$ arc lamps, and air-tovacuum and heliocentric corrections were applied. The spectrum covers the range of $0.39-1.05 \mu \mathrm{m}$ at a resolution of $11.5 \mathrm{~km} \mathrm{~s}^{-1}$. 


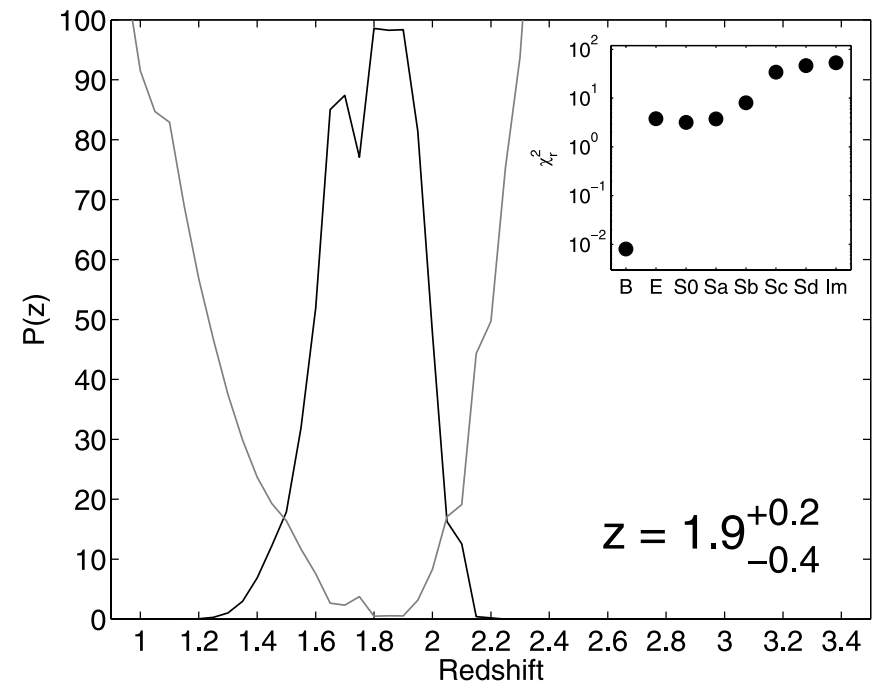

FIG. 2.-Probability distribution (black line) and reduced $\chi^{2}$ (gray line) for the host galaxy photometric redshift calculated with the hyperz software package (Bolzonella et al. 2000) using a starburst template. The best-fit redshift is $z=$ $1.9_{-0.4}^{+0.2}$. The inset shows the reduced $\chi^{2}$ for the best-fit solution using the various galaxy templates. Clearly, the starburst template provides the only adequate fit to the data.

We detect continuum emission beyond $0.6 \mu \mathrm{m}$, but no emission or absorption lines are clearly identified.

A $5400 \mathrm{~s}$ spectrum of the host was obtained with the Low Resolution Imaging Spectrometer (LRIS) on the Keck I $10 \mathrm{~m}$ telescope on 2004 April 22 UT. The wavelength coverage is 0.36 to $0.96 \mu \mathrm{m}$, with a resolution of $3.3 \AA(0.36-0.58 \mu \mathrm{m})$ and $5.6 \AA(0.58-$ $0.96 \mu \mathrm{m})$. The data were reduced as described above, and flux calibration was performed relative to the spectrophotometric standard Feige 34. As in the ESI spectra, we detect the host continuum but no emission or absorption features in the range of $0.6-0.96 \mu \mathrm{m}$.

The limit on line emission redward of about $0.9 \mu \mathrm{m}$ is $F \lesssim 2 \times$ $10^{-17} \mathrm{erg} \mathrm{cm}^{-2} \mathrm{~s}^{-1}$ (integrated over $10 \AA$ ) , corresponding to a limit of $L([\mathrm{O}$ I $]) \lesssim 3 \times 10^{41}$ at $z=1.55$ (the redshift limit for the detection of $\mathrm{O}$ II 23727 in our spectrum). This corresponds to a limit on the star formation rate of $\lesssim 4 M_{\odot} \mathrm{yr}^{-1}$ (Kennicutt 1998). We note that blueward of $0.9 \mu \mathrm{m}$ the limits are even deeper due to the correspondingly lower redshift of a putative $\mathrm{O}$ II line and lower noise level of our spectrum. We therefore conclude that the lack of $\mathrm{O}_{\text {II l}}$ line emission indicates a host redshift of $z>1.55$. As we show in $\S 3$, this limit is fully consistent with our photometric redshift and star formation rate estimates.

\section{HOST GALAXY PROPERTIES}

The optical/NIR spectral energy distribution (SED) of the host galaxy is shown in Figure 1. With $R-K_{s}=6.2 \pm 0.2 \mathrm{mag}$, the host is classified as an ERO and is the reddest GRB host to date. EROs fall in two general categories of old, passively evolving elliptical galaxies and dust-obscured star-forming galaxies (McCarthy 2004). Long GRBs have never been localized to elliptical galaxies, and we further distinguish the two possibilities using the observed SED. We use the hyperz package (Bolzonella et al. 2000) to fit a range of model templates (starburst, E, S0-Sc, and Irr) with the redshift, stellar population age, and extinction as free parameters. We assume a Calzetti et al. (2000) extinction curve. The elliptical galaxy model provides an unsatisfactory fit, with a probability of only $0.7 \%\left(\chi_{r}^{2}=4.1\right.$; Figs. 1 and 2$)$, due to the expected flat slope (in $F_{\lambda}$ ) beyond $1 \mu \mathrm{m}$ and a brighter $g^{\prime}$-band magnitude.

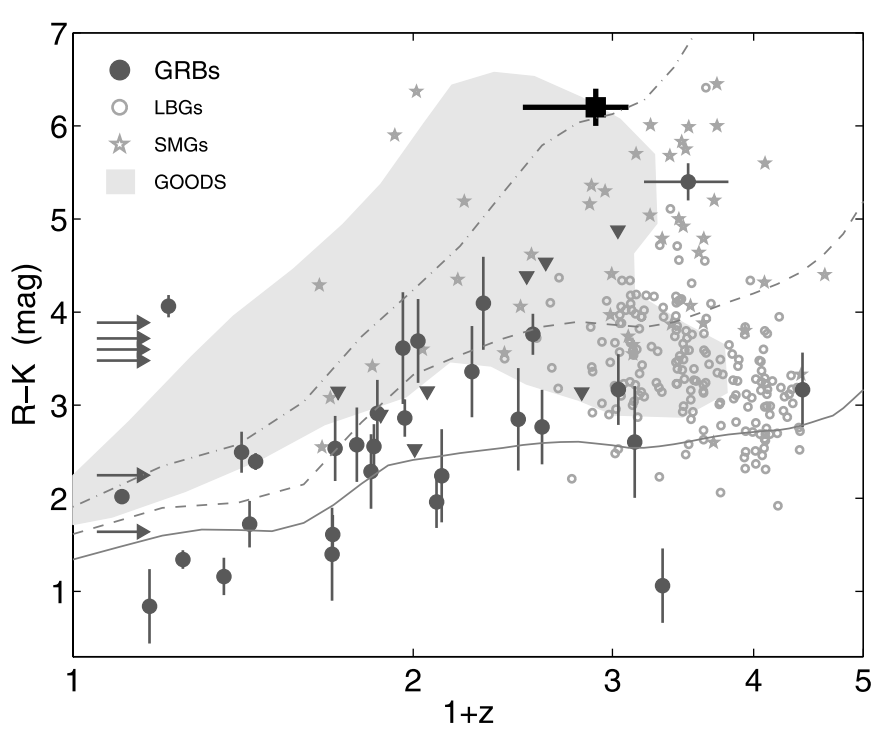

Fig. 3.-Apparent $R-K_{s}$ color plotted vs. redshift for GRB host galaxies ( filled circles; Chary et al. 2002; Le Floc'h et al. 2003; Berger et al. 2003; Levan et al. 2006), LBGs (open circles; Shapley et al. 2001; Steidel et al. 2004), submillimeter galaxies (stars; Smail et al. 2004), and GOODS galaxies (shaded; Somerville et al. 2004). Arrows designate GRB hosts without a measured redshift. The host galaxy of GRB 020127 (black square) is significantly redder than the typical color of $R-K_{s} \approx 2.5-3 \mathrm{mag}$. The gray lines are tracks of $R-K_{s}$ color as a function of redshift from the population synthesis code of Bruzual \& Charlot (2003) for a stellar population age of $0.1 \mathrm{Gyr}$ (solid), $0.3 \mathrm{Gyr}$ (dashed), and $1 \mathrm{Gyr}$ (dot-dashed). The majority of the GRB hosts appear to have ages of $\sim 0.1-0.3 \mathrm{Gyr}$. These young stellar population ages, and not metallicity, may be the underlying reason for the blue colors and low luminosities of GRB hosts.

The starburst template, on the other hand, provides an excellent fit $\left(P_{\max }=99.9 \%\right.$; Fig. 2). The best-fit parameters are $z=1.9_{-0.4}^{+0.2}$, an age of about $0.7 \mathrm{Gyr}$, a rest-frame extinction $A_{V} \approx 0.5 \mathrm{mag}$, and an absolute rest-frame magnitude $M_{\mathrm{AB}}(B)=-23.5 \pm 0.1 \mathrm{mag}$. This corresponds to $L \approx 5 L^{*}$ (Dahlen et al. 2005; Willmer et al. 2006). A comparison to UV-selected galaxies at $z \sim 2$ (Shapley et al. 2005) indicates that the host mass is $M_{*} \sim 10^{11}-10^{12} M_{\odot}$. Using the relation of Kennicutt (1998) and the observed $R$-band (2200 ̊ rest-frame) flux of $0.46 \mu \mathrm{Jy}$, we estimate an unobscured star formation rate of $\sim 6 M_{\odot} \mathrm{yr}^{-1}$. The value corrected for extinction is about 1 order of magnitude larger.

The host of GRB 020127 is distinguished from other GRB host galaxies in several ways. First, it is more than 3 mag redder than the average value for GRB hosts, and even 1 mag redder than the host of GRB 030115 (Fig. 3). This is due to the combined effect of extinction, and a more evolved stellar population compared to the typical value of about $0.1 \mathrm{Gyr}$ for other GRB hosts (Christensen et al. 2004). Second, it is 4 mag more luminous than the median value of $M_{\mathrm{AB}}(B) \approx-19.5 \mathrm{mag}$ for GRB hosts (with a range of -16 to $-22 \mathrm{mag}$; Fig. 4 ). Third, the inferred stellar mass is nearly 2 orders of magnitude larger than the median value of $10^{9.5} M_{\odot}$ for GRB hosts (Christensen et al. 2004; Savaglio et al. 2006). Finally, the unobscured specific star formation rate of about $1 M_{\odot} \mathrm{yr}^{-1}$ $(L / L *)^{-1}$, is nearly 1 order of magnitude lower than for other GRB hosts (Christensen et al. 2004), but we note that with the extinction correction it is in fact similar.

Perhaps most importantly, a comparison to the mass-metallicity relation of UV-selected galaxies at $z \sim 2$ (Erb et al. 2006) indicates that the host of GRB 020127 has a high metallicity, in the range of about $0.5-1 Z_{\odot}$. This is also similar to the metallicities of the so-called distant red galaxies (DRGs; $J-K>2.3 \mathrm{mag}$ ), which have median ages and masses of $\sim 2$ Gyr and $\sim 10^{11} M_{\odot}$, 


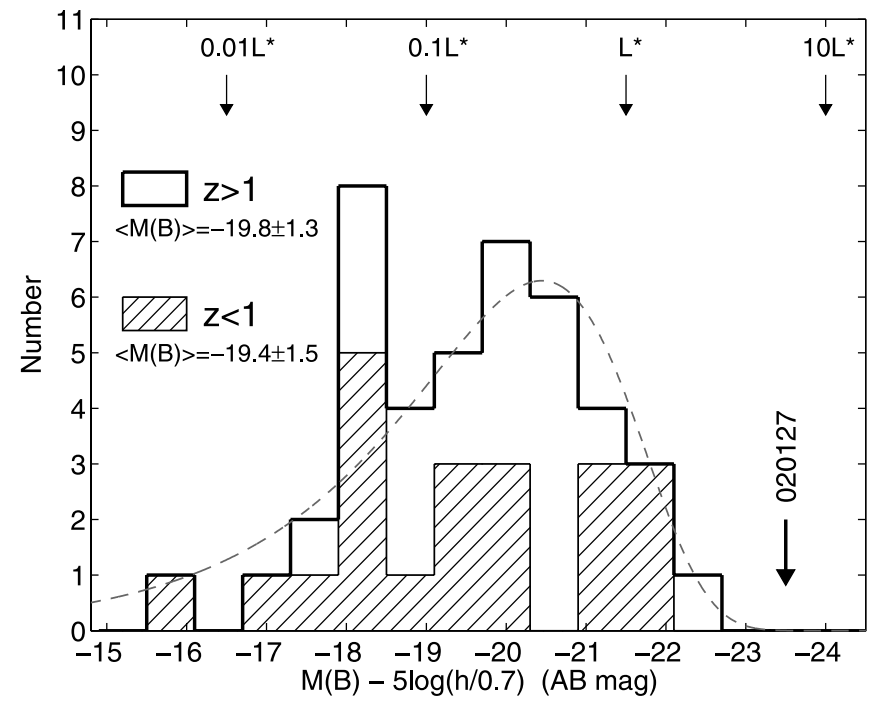

FIG. 4.-Distribution of absolute rest-frame $B$-band magnitudes for GRB host galaxies (Berger et al. 2007, in preparation). The median value for the sample is $M_{\mathrm{AB}}(B)=-19.5 \mathrm{mag}$, or $0.1 L^{*}$, and it does not appear to significantly vary between $z<1$ and $z>1$ (values in the figure are the median and standard deviation). GRB 020127 clearly stands out as the most luminous, and thus most massive host galaxy detected to date, but we note that the overall luminosity distribution is in good agreement with that of other high-redshift galaxy samples (see also Jakobsson et al. 2005). The dashed curve is a representative Schechter luminosity function with $M_{B}^{*}=-21 \mathrm{mag}$ and $\alpha=-1.4$.

respectively (Förster Schreiber et al. 2004; van Dokkum et al. 2004).

\section{DISCUSSION}

The host galaxy of GRB 020127, identified through X-ray and radio afterglow observations, is the reddest, and most luminous and massive, GRB host discovered to date. Unfortunately, the early optical limits $(\S 2)$ are too shallow to ascertain whether the afterglow was significantly extinguished by dust; from the X-ray flux at 4.14 days we expect $R \sim 21$ mag at the time of the early optical observations, at the level of the available limits. For GRB 030115, the only other burst with an ERO host galaxy, the afterglow itself was shown to be significantly dust extinguished, with $R-K \approx 6$ mag (Levan et al. 2006). It is therefore possible that dusty galaxies are underrepresented in the current GRB host sample, due to obscuration of the optical afterglow, but we stress that the hosts of several dark bursts are not significantly redder than the median of the sample (Berger et al. 2003).

The discovery of a GRB in a massive, and hence metal-enriched, starburst galaxy indicates that the proposed low-metallicity bias of GRB progenitors $\left(\lesssim 0.1 Z_{\odot}\right.$; Fruchter et al. 2006; Stanek et al. 2006) is not likely to be as severe as previously claimed, particularly at $z \gtrsim 1$. This appears to be true not only in terms of an absolute metallicity cutoff, but also relative to the mass-metallicity relation of galaxies at high redshift. In particular, while the hosts of GRBs at $z \lesssim 0.2$ appear to have metallicities at the low end of the distribution for local galaxies (Stanek et al. 2006), at least some GRBs at $z \sim 2$ occur in the most metal-enriched galaxies at that redshift. This conclusion is also supported by the metallicities derived from afterglow absorption spectra (at $z>2$ ), which range up to solar values (Berger et al. 2006a; Prochaska 2006), as well as by the detection of submillimeter emission from some GRB hosts (Berger et al. 2003). Since GRB progenitors appear to occur in galaxies with metallicities at least up to $\sim 0.5 Z_{\odot}$, and given that the $M-Z$ relations found at $z \sim 1-2$ are systematically lower by about $0.3 \mathrm{dex}$ compared to the local relation (Tremonti et al. 2004; Savaglio et al. 2005; Erb et al. 2006), we conclude that even if a slight lowmetallicity bias does exist, its effect will diminish beyond $z \sim 1$.

Instead, we suggest that the blue colors and low masses of GRB host galaxies may reflect their young stellar populations. This is likely related to the fact that GRB progenitors are massive stars, which explode within a few million years of formation, thereby leading to the selection of young starburst galaxies. In fact, for $z \sim 1-2$, the average $R-K$ color of a 0.1 Gyr population is about $2.5 \mathrm{mag}$, in good agreement with the observed colors of GRB hosts, while for 0.3 and 1 Gyr populations it is about 1 and 2.5 mag redder, respectively (Fig. 3).

This explanation also accounts for the transition at low redshift $(z \lesssim 0.2)$ from a representative to a predominantly low-luminosity host population, since locally starburst activity occurs primarily in low-mass galaxies. Brinchmann et al. (2004) show that the local fraction of galaxies with recent starburst activity increases strongly with decreased mass. Similarly, Bell et al. (2005) show that while at $z \sim 0.7$ nearly half of all galaxies with $M \gtrsim 10^{10} M_{\odot}$ undergo intense star formation activity, locally this number is less than $1 \%$. This is essentially a reflection of galaxy "downsizing"; the transition of star formation activity from high-mass to low-mass (and hence low-metallicity) galaxies as a function of decreasing redshift, as noted in part by the earlier transition from star formation to quiescence for massive galaxies (Juneau et al. 2005), and the downward shift of the typical mass $\left(M^{*}\right)$ of blue galaxies with decreasing redshift (Borch et al. 2006; Bundy et al. 2006).

In our scenario, therefore, the driving parameter is star formation activity and stellar population age, which may be misidentified as a low-metallicity bias at low redshift. Thus, we conclude that GRBs and their host galaxies are likely to trace star formation in a relatively unbiased way, with possibly a preference for younger starburst populations. If this is in fact the case, the overall luminosity function of GRB host galaxies (see also Jakobsson et al. 2005) indicates that the bulk of the star formation at $z \gtrsim 1$ takes place in galaxies fainter than $L^{*}$, in good agreement with the steep faint-end slope of the luminosity function of high-redshift galaxies.

We thank J. Kollmeier, D. Erb, and the anonymous referee for helpful comments on the manuscript. E. B. is supported by NASA through Hubble Fellowship grant HST-01171.01 awarded by STScI, which is operated by AURA, Inc. for NASA under contract NAS5-26555. The data presented herein were obtained at the W. M. Keck Observatory, which is operated as a scientific partnership among the California Institute of Technology, the University of California, and the National Aeronautics and Space Administration. The Observatory was made possible by the generous financial support of the W. M. Keck Foundation.
Barnard, V. E., et al. 2003, MNRAS, 338, 1

Bell, E. F., et al. 2005, ApJ, 625, 23

Berger, E., Cowie, L. L., Kulkarni, S. R., Frail, D. A., Aussel, H., \& Barger, A. J. 2003, ApJ, 588, 99

Berger, E., Penprase, B. E., Cenko, S. B., Kulkarni, S. R., Fox, D. B., Steidel, C. C., \& Reddy, N. A. 2006a, ApJ, 642, 979

\section{REFERENCES}

Berger, E., et al. 2006b, ApJ, submitted (astro-ph/0603689)

Bloom, J. S., Kulkarni, S. R., \& Djorgovski, S. G. 2002, AJ, 123, 1111

Bolzonella, M., Miralles, J.-M., \& Pelló, R. 2000, A\&A, 363, 476

Borch, A., et al. 2006, A\&A, 453, 869

Brinchmann, J., Charlot, S., White, S. D. M., Tremonti, C., Kauffmann, G., Heckman, T., \& Brinkmann, J. 2004, MNRAS, 351, 1151 
Bruzual, G., \& Charlot, S. 2003, MNRAS, 344, 1000

Bundy, K., et al. 2006, ApJ, 651, 120

Calzetti, D., Armus, L., Bohlin, R. C., Kinney, A. L., Koornneef, J., \& StorchiBergmann, T. 2000, ApJ, 533, 682

Castro Cerón, J. M., Gorosabel, J., Greiner, J., Klose, S., Snigula, J., \& CastroTirado, A. J. 2002, GCN Circ., 1234, http://gcn.gsfc.nasa.gov/gen3/1234.gen3

Chary, R., Becklin, E. E., \& Armus, L. 2002, ApJ, 566, 229

Christensen, L., Hjorth, J., \& Gorosabel, J. 2004, A\&A, 425, 913

Dahlen, T., Mobasher, B., Somerville, R. S., Moustakas, L. A., Dickinson, M., Ferguson, H. C., \& Giavalisco, M. 2005, ApJ, 631, 126

Erb, D. K., Shapley, A. E., Pettini, M., Steidel, C. C., Reddy, N. A., \& Adelberger, K. L. 2006, ApJ, 644, 813

Förster Schreiber, N. M., et al. 2004, ApJ, 616, 40

Fruchter, A. S., \& Hook, R. N. 2002, PASP, 114, 144

Fruchter, A. S., et al. 2006, Nature, 441, 463

Fynbo, J. P. U., et al. 2003, A\&A, 406, L63

Heger, A., Fryer, C. L., Woosley, S. E., Langer, N., \& Hartmann, D. H. 2003, ApJ, 591, 288

Jakobsson, P., et al. 2005, MNRAS, 362, 245

Juneau, S., et al. 2005, ApJ, 619, L135

Kelson, D. D. 2003, PASP, 115,688

Kennicutt, R. C., Jr. 1998, ARA\&A, 36, 189

Kochanek, C. S., et al. 2001, ApJ, 560, 566

Lamb, D. Q., York, D. G., McMillan, R. J., \& Johnston, D. 2002, GCN Circ., 1230, http://gcn.gsfc.nasa.gov/gen3/1230.gen3

Le Floc'h, E., Charmandaris, V., Forrest, W. J., Mirabel, I. F., Armus, L., \& Devost, D. 2006, ApJ, 642, 636
Le Floc'h, E., et al. 2003, A\&A, 400, 499

Levan, A., et al. 2006, ApJ, 647, 471

MacFadyen, A. I., \& Woosley, S. E. 1999, ApJ, 524, 262

McCarthy, P. J. 2004, ARA\&A, 42, 477

Prochaska, J. X. 2006, ApJ, 650, 272

Ricker, G., et al. 2002, GCN Circ., 1229, http://gcn.gsfc.nasa.gov/gcn3/1229.gcn3 Sakamoto, T., et al. 2005, ApJ, 629, 311

Savaglio, S., Glazebrook, K., \& Le Borgne, D. 2006, in AIP Conf. Proc. 836, GRB Host Studies (GHostS), ed. S. S. Holt, N. Gehrels, \& J. A. Nousek (New York: AIP), 540

Savaglio, S., et al. 2005, ApJ, 635, 260

Schlegel, D. J., Finkbeiner, D. P., \& Davis, M. 1998, ApJ, 500, 525

Shapley, A. E., Steidel, C. C., Adelberger, K. L., Dickinson, M., Giavalisco, M., \& Pettini, M. 2001, ApJ, 562, 95

Shapley, A. E., Steidel, C. C., Erb, D. K., Reddy, N. A., Adelberger, K. L., Pettini, M., Barmby, P., \& Huang, J. 2005, ApJ, 626, 698

Smail, I., Chapman, S. C., Blain, A. W., \& Ivison, R. J. 2004, ApJ, 616, 71 Somerville, R. S., et al. 2004, ApJ, 600, L135

Stanek, K. Z., et al. 2006, Acta Astron., 56, 333

Steidel, C. C., Shapley, A. E., Pettini, M., Adelberger, K. L., Erb, D. K., Reddy, N. A., \& Hunt, M. P. 2004, ApJ, 604, 534

Tremonti, C. A., et al. 2004, ApJ, 613, 898

van Dokkum, P. G., et al. 2004, ApJ, 611, 703

Willmer, C. N. A., et al. 2006, ApJ, 647, 853 